\title{
A quadratic stability result for singular switched systems with application to anti-windup control
}

\author{
R. Shorten ${ }^{1 *}$ M. Corless ${ }^{2 \dagger}$, R. Middleton ${ }^{1}$, S. Klinge ${ }^{1}$, K. Wulff $^{3 \ddagger}$
}

\begin{abstract}
In this note we consider the problem of determining necessary and sufficient conditions for the existence of a common quadratic Lyapunov function for a pair of stable linear time-invariant systems whose system matrices are of the form $A, A-g h^{T}$, and where one of the matrices is singular. We then apply this result in a study of a feedback system with a saturating actuator.
\end{abstract}

\section{Introduction}

Consider a switching system described by

$$
\dot{x}=\left[A-\sigma(t, x) g h^{T}\right] x
$$

where the state $x(t)$ and $g, h$ are real vectors, $A$ is a real square matrix, and the scalar switching function $\sigma$ satisfies

$$
0 \leq \sigma(t, x) \leq 1
$$

Suppose $A$ is a Hurwitz matrix, that is, all its eigenvalues have negative real parts; then the system corresponding to $\sigma(t, x) \equiv 0$, that is, $\dot{x}=A x$, is globally asymptotically stable about the origin of the state space. Suppose also that all the eigenvalues of $A-g h^{T}$ have

${ }^{*} 1$. The Hamilton Institute, NUI Maynooth, Ireland. Work supported under Science Foundation of Ireland grants: SFI 07/RPR/I177

†2. School of Aeronautics \& Astronautics, Purdue University, West Lafayette, IN, USA

†3. Fachgebiet Regelungstechnik, TU Ilmenau, Germany negative real parts except for a single eigenvalue at zero. Then the system corresponding to $\sigma(t, x) \equiv 1$, that is, $\dot{x}=\left(A-g h^{T}\right) x$, is stable (but not asymptotically stable) about the origin and all its solutions are bounded. We can guarantee that the switching system (1) is stable about the origin and all solutions are bounded if there is a real symmetric positive definite matrix $P$ satisfying the following two Lyapunov matrix inequalities.

$$
\begin{array}{r}
A^{T} P+P A<0 \\
\left(A-g h^{T}\right)^{T} P+P\left(A-g h^{T}\right) \leq 0 .
\end{array}
$$

We refer to a matrix $P=P^{T}>0$ satisfying (3) as a common Lyapunov matrix for $A$ and $A-g h^{T}$, and $V(x):=x^{T} P x$ is referred to as a common quadratic Lyapunov function.

Such stability problems arise in many applications [1, 2, 3, 4] and one such application shall be discussed later in this note. Our principal theoretical result here is to show that the following elementary condition is both necessary and sufficient for the existence of a common Lyapunov matrix $P$.

The matrix product $A\left(A-g h^{T}\right)$ has no negative real eigenvalues and only one zero eigenvalue.

\section{Strictly positive real transfer functions}

In this section we present some results on strictly positive real transfer functions which are useful in the development of the main result. In particular, Theorem 2.1 provides a simple spectral characterization of strictly positive real transfer functions. Due to space 
limitations, these results are all given without proof; proofs are available in the journal submission [5] or [6, Appendix B].

In what follows, $A$ is a real $n \times n$ matrix and $b, c, g, h$ are real $n$-vectors. Recall that a scalar transfer function $H$ is strictly positive real $(S P R)$ if there exists a scalar $\alpha>0$ such that $H$ is analytic in a region of the complex plane for which $\operatorname{Re}(s) \geq-\alpha$ and

$$
H(j \omega-\alpha)+H(j \omega-\alpha)^{*} \geq 0 \text { for all } \omega \in \mathbb{R} .
$$

We assume throughout that $H$ is not identically zero. The following standard result provides a more convenient characterization of SPR.

Lemma 2.1 [7] Suppose A is Hurwitz. Then the transfer function $H(s)=c^{T}(s I-A)^{-1} b$ is $S P R$ if and only if for all $\omega \in \mathbb{R}$

$$
\begin{aligned}
H(j \omega)+H(j \omega)^{*} & >0 \\
\lim _{\omega \rightarrow \infty} \omega^{2}\left[H(j \omega)+H(j \omega)^{*}\right] & >0 .
\end{aligned}
$$

In checking SPR of a system it is sometimes more convenient to first transform the system to an equivalent (from an SPR viewpoint) form. The following lemma provides such an equivalent system.

Lemma 2.2 [5] The transfer function $H(s)=$ $c^{T}(s I-A)^{-1} b$ is SPR if and only if $H_{I}(s)=$ $c^{T}\left(s I-A^{-1}\right)^{-1} b$ is SPR.

The core of our main result is based on a spectral condition for strict positive realness [8], [9], [10]. This result follows as an immediate consequence of the following lemma.

Lemma $2.3[11,12,13]$ Let $H(s)=d+$ $c^{T}(s I-A)^{-1} b$ where $A$ is invertible. Then, $H\left(s^{-1}\right)=\bar{d}+\bar{c}^{T}(s I-\bar{A})^{-1} \bar{b}$ with $\bar{A}=A^{-1}$, $\bar{b}=-A^{-1} b, \bar{c}^{T}=c^{T} A^{-1}$ and $\bar{d}=d-c^{T} A^{-1} b$.

Comment : Note that when $H$ is SPR we must have $\bar{d}>0$. This follows from the fact that $\bar{d}=H(0)$ and $H(0)+H(0)^{*}>0$ since $H$ is SPR.

Now we give the aforementioned spectral characterisation of strict positive realness [5].
Theorem 2.1 Suppose A is Hurwitz. Then, the following statements are equivalent.

(a) The transfer function $H(s)=c^{T}(s I-$ $A)^{-1} b$ is SPR.

(b) $c^{T} A^{-1} b<0$ and the matrix product $A^{-1}\left(A^{-1}-\frac{A^{-1} b c^{T} A^{-1}}{c^{T} A^{-1} b}\right)$ has no negative real eigenvalues and exactly one zero eigenvalue.

(c) $c^{T} A b<0$ and the matrix product $A(A-$ $\left.\frac{A b c^{T} A}{c^{T} A b}\right)$ has no negative real eigenvalues and exactly one zero eigenvalue.

\section{Main result}

Our main result makes use of the following observations. First note that $P=P^{T}>0$ is a strict Lyapunov matrix for $A$, that is,

$$
A^{T} P+P A<0
$$

if and only if $P$ is a strict Lyapunov matrix for $A^{-1}$, that is,

$$
A^{-T} P+P A^{-1}<0
$$

To see, this post- and pre-multiply the first inequality by $A^{-1}$ and its transpose. In a similar fashion one can also show that $P$ is a (nonstrict) Lyapunov matrix for $A$, that is,

$$
A^{T} P+P A \leq 0
$$

if and only if $P$ is a (non-strict) Lyapunov matrix for $A^{-1}$, that is,

$$
P A^{-1}+A^{-T} P \leq 0
$$

We now present the main result, the proof of which requires the following KYP lemma.

Lemma 3.1 [14] Suppose $(A, b)$ is controllable and $(A, c)$ is observable. Then, the following statements are equivalent.

(i) The matrix $A$ is Hurwitz and the transfer function $H(s)=c^{T}(s I-A)^{-1} b$ is SPR. 
(ii) There exists a matrix $P=P^{T}>0$ that satisfies the constrained Lyapunov inequality:

$$
\begin{aligned}
A^{T} P+P A & <0 \\
P b & =c .
\end{aligned}
$$

(iii) There exists a matrix $P=P^{T}>0$ such that the following Lyapunov inequalities are satisfied:

$$
\begin{aligned}
A^{T} P+P A & <0 \\
-\left(c b^{T} P+P b c^{T}\right) & \leq 0 .
\end{aligned}
$$

Comment: The assumption that $(A, c)$ is observable ensures that $P$ is positive definite in the theorem [15].

Theorem 3.1 (Main Theorem) Suppose that $A$ is Hurwitz and all the eigenvalues of $A-g h^{T}$ have negative real part, except one, which is zero. Suppose also that $(A, g)$ is controllable and $(A, h)$ is observable. Then, there exists a matrix $P=P^{T}>0$ such that

$$
\begin{array}{r}
A^{T} P+P A<0 \\
\left(A-g h^{T}\right)^{T} P+P\left(A-g h^{T}\right) \leq 0
\end{array}
$$

if and only if the matrix product $A\left(A-g h^{T}\right)$ has no real negative eigenvalues and exactly one zero eigenvalue.

Proof: The proof consists of two parts. First we use an equivalence to show that the conditions on $A\left(A-g h^{T}\right)$ are sufficient for the existence of a Lyapunov matrix $P$ with the required properties. We then show that these conditions are also necessary.

Sufficiency: Let $c=A^{-T} h$ and let $b$ be a right eigenvector of $A-g h^{T}$ corresponding to the zero eigenvalue. Then $b \neq 0, A b=g h^{T} b=$ $h^{T} b g$ and $c^{T} A b=h^{T} b$. Since $A$ is Hurwitz, we must have $h^{T} b \neq 0$, otherwise $A b=0$. Hence $c^{T} A b \neq 0$ and, without loss of generality, we assume that $b$ is chosen so that $c^{T} A b=-1$. In this case,

$$
g=-A b \quad \text { and } \quad h^{T}=c^{T} A .
$$

Controllability of $(A, b)$ and observability of $(A, c)$ follow from controllability of $(A, g)$ and observability of $(A, h)$, respectively.

Noting that

$$
A_{2}:=A-g h^{T}=A-\frac{A b c^{T} A}{c^{T} A b},
$$

it follows from Theorem 2.1 that the conditions on $A A_{2}$ imply that the transfer function $c^{T}(s I-A)^{-1} b$ is SPR. Consequently, it follows from Lemma 3.1 that there exists a matrix $P=P^{T}>0$ such that

$$
\begin{array}{r}
A^{T} P+P A<0 \\
P b=c .
\end{array}
$$

Pre- and post- multiplying the above inequality by $A^{-T}$ and $A^{-1}$ shows that this inequality is equivalent to

$$
A^{-T} P+P A^{-1}<0
$$

This last inequality and (11) imply that

$$
\left[\begin{array}{cc}
A^{-T} P+P A^{-1} & P b-c \\
b^{T} P-c^{T} & 0
\end{array}\right] \leq 0 .
$$

Hence,

$$
\begin{gathered}
{\left[\begin{array}{cc}
A^{-T} & -c \\
b^{T} & 0
\end{array}\right]\left[\begin{array}{ll}
P & 0 \\
0 & 1
\end{array}\right]} \\
+\left[\begin{array}{ll}
P & 0 \\
0 & 1
\end{array}\right]\left[\begin{array}{cc}
A^{-1} & b \\
-c^{T} & 0
\end{array}\right] \leq 0 .
\end{gathered}
$$

Since $c^{T} A b=-1 \neq 0$,

$$
\begin{aligned}
{\left[\begin{array}{cc}
A^{-1} & b \\
-c^{T} & 0
\end{array}\right]^{-1} } & =\left[\begin{array}{cc}
A-\frac{A b c^{T} A}{c^{T} A b} & -\frac{A b}{c^{T} A b} \\
\frac{c^{T} A}{c^{T} A b} & \frac{1}{c^{T} A b}
\end{array}\right] \\
& =\left[\begin{array}{cc}
A-g h^{T} & -g \\
-h^{T} & -1
\end{array}\right],
\end{aligned}
$$

and consequently that

$$
\left[\begin{array}{cc}
\left(A-g h^{T}\right)^{T} P+P\left(A-g h^{T}\right) & -P g-h \\
-g^{T} P-h^{T} & -2
\end{array}\right] \leq 0 .
$$

It immediately follows that for the above inequality to hold, we must have

$$
\left(A-g h^{T}\right)^{T} P+P\left(A-g h^{T}\right) \leq 0 .
$$


Necessity : We first show that if there exists a matrix $P=P^{T}>0$ satisfying conditions (8)-(9), then $A A_{2}$ cannot have a negative real eigenvalue. Note that the conditions on $P$ are equivalent to

$$
\begin{aligned}
A^{-T} P+P A^{-1} & <0 \\
A_{2}^{T} P+P A_{2} & \leq 0
\end{aligned}
$$

Hence, for any $\gamma>0$,

$$
\left(A_{2}+\gamma A^{-1}\right)^{T} P+P\left(A_{2}+\gamma A^{-1}\right)<0 .
$$

Since $P=P^{T}>0$ this Lyapunov inequality implies that $A_{2}+\gamma A^{-1}$ must be Hurwitz and hence, non-singular. Thus $A A_{2}+\gamma I$ is nonsingular for all $\gamma>0$. This means that $A A_{2}$ cannot have a negative real eigenvalue [8].

We now show that $A A_{2}$ cannot have a zero eigenvalue whose multiplicity is greater that one. To this end introduce the matrix

$$
\tilde{A}(k)=A_{2}+k g h^{T} .
$$

Then $A=\tilde{A}(1)$ and inequalities (8)-(9) hold if and only if

$$
\begin{array}{r}
\tilde{A}(k)^{T} P+P \tilde{A}(k)<0 \\
A_{2}^{T} P+P A_{2} \leq 0
\end{array}
$$

hold for all $k$ sufficiently close to one. As we have seen above, this implies that $A(k) A_{2}$ cannot have negative real eigenvalues for all $k$ sufficiently close to one. We shall show that $A A_{2}$ having an eigenvalue at the origin whose multiplicity is greater than one contradicts this statement.

By assumption, $A_{2}$ has a single eigenvalue at zero; a corresponding eigenvector is the vector $b$. Clearly, $b$ is also an eigenvector corresponding to a zero eigenvalue of $A(k) A_{2}$. Now choose any nonsingular matrix $T$ whose first column is $b$. Then,

$$
T^{-1} A(k) A_{2} T=\left(\begin{array}{cc}
0 & * \\
0 & S+k r s^{T}
\end{array}\right)
$$

and the eigenvalues of $A(k) A_{2}$ consist of zero and the eigenvalues of $S+k r s^{T}$. Note that the matrix $S$ must be invertible since

$$
T^{-1} A_{2}^{2} T=T^{-1} A(0) A_{2} T=\left(\begin{array}{cc}
0 & * \\
0 & S
\end{array}\right)
$$

and $A_{2}^{2}$ has only a single eigenvalue at zero. Now suppose that $A A_{2}=A(1) A_{2}$ has an eigenvalue at the origin whose multiplicity is greater than one. Then $S+r d^{T}$ must have a eigenvalue at zero; hence, $\operatorname{det}\left[S+r s^{T}\right]=0$. Since $S$ is invertible,

$$
\begin{aligned}
\operatorname{det}\left[S+k r s^{T}\right]= & \operatorname{det}[S] \operatorname{det}\left[I+k S^{-1} r s^{T}\right] \\
& =\operatorname{det}[S]\left(1+k s^{T} S^{-1} r\right),
\end{aligned}
$$

and we must have $1+s^{T} S^{-1} r=0$ which implies that $s^{T} S^{-1} r=-1$. Hence,

$$
\operatorname{det}\left[S+k r s^{T}\right]=\operatorname{det}[S](1-k) \text {. }
$$

Suppose $\operatorname{det}[S]>0$. Then,

$$
\operatorname{det}\left[S+k r s^{T}\right]<0
$$

for $k>1$. Since $\operatorname{det}\left[S+k r s^{T}\right]$ is the product of all the eigenvalues of $S+k r s^{T}$ and complex eigenvalues occur in complex conjugate pairs, $S+k r s^{T}$ must have at least one real negative eigenvalue when $k>1$. This yields the contradiction that $A(k) A_{2}$ has a negative real eigenvalue when $k>1$. The conclusion is the same for $\operatorname{det}[S]<0$.

\section{Application to an Example Antiwindup Control of an Integrating Process}

One of the applications for the results above arises in the context of Anti-windup control of integrating processes that arises in many contexts, including control of platoons of vehicles $[6, \S 3.2]$. One of the popular schemes for Anti-windup control uses feedback of the error between the actual (saturated) control signal and the control signal that would have been commanded in the absence of saturation, as illustrated in Figure 1.

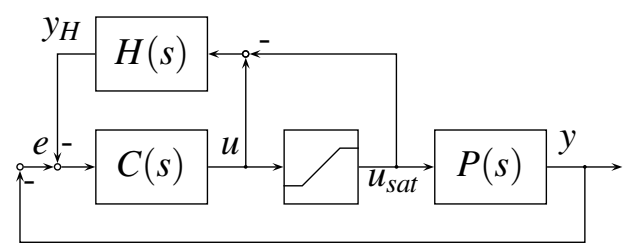

Figure 1: Anti-windup scheme 
For simplicity we take the saturation function to be symmetric, that is, with $u_{\max }>0$

$u_{\text {sat }}=\phi(u):=\left\{\begin{array}{cc}u & \text { for }|u| \leq u_{\max } \\ u_{\max } \operatorname{sgn}(u) & \text { else }\end{array}\right\}$.

Define $k_{\phi}$ as

$$
k_{\phi}(u):=\left\{\begin{array}{cc}
1 & \text { for }|u| \leq u_{\max } \\
u_{\max } /|u| & \text { else }
\end{array}\right\}
$$

In this case, consider state space realizations for $P(s), C(s), H(s)$ as $\left(A_{p}, B_{p}, C_{p}\right)$, $\left(A_{c}, B_{c}, C_{c}, D_{c}\right)$ and $\left(A_{h}, B_{h}, C_{h}\right)$ respectively. From the parameter values given in [6] we obtain:

$$
\begin{array}{cc}
A_{p}=\left[\begin{array}{cc}
0 & 1 \\
0 & -0.021
\end{array}\right] ; & B_{p}=\left[\begin{array}{l}
0 \\
1
\end{array}\right] \\
C_{p}=\left[\begin{array}{ll}
1 & 0
\end{array}\right], & \\
A_{c}=\left[\begin{array}{cc}
0 & 0 \\
0 & -30
\end{array}\right] ; & B_{c}=\left[\begin{array}{c}
0.17 \\
1
\end{array}\right] \\
C_{c}=\left[\begin{array}{ll}
1 & -3690
\end{array}\right] & D_{c}=[124.67]
\end{array}
$$

and

$$
\begin{aligned}
A_{h} & =\left[\begin{array}{ccc}
-0.021 & 1.674 & 0.307 \\
0 & -0.200 & 5.459 \\
0 & 0 & -0.200
\end{array}\right], \\
B_{h} & =\left[\begin{array}{lll}
0 & 0 & 0.125
\end{array}\right]^{T}, \\
C_{h} & =\left[\begin{array}{lll}
0.0074 & 0.1310 & 0.0240
\end{array}\right]
\end{aligned}
$$

In this case, the overall equations for the system of Figure 1 can be written by taking the combined state, $x=\left[\begin{array}{lll}x_{p}^{T} & x_{c}^{T} & x_{h}^{T}\end{array}\right]^{T}$ and have the form (1) where

$$
\begin{gathered}
A=\left[\begin{array}{ccc}
A_{p}-B_{p} D_{c} C_{p} & B_{p} C_{c} & -B_{p} D_{c} C_{h} \\
-B_{c} C_{p} & A_{c} & -B_{c} C_{h} \\
0 & 0 & A_{h}
\end{array}\right], \\
g^{T}=\left[\begin{array}{lll}
B_{p}^{T} & 0 & -B_{h}^{T}
\end{array}\right], \\
h^{T}=\left[\begin{array}{lll}
-D_{c} C_{p} & C_{c} & -D_{c} C_{h}
\end{array}\right]
\end{gathered}
$$

and the switching function is given by

$$
\sigma(t, x)=1-k_{\phi}(u) \in[0,1]
$$

for $u=h^{T} x$.

Note that by analysis of the specific matrices in this example, we can show that indeed, $A$ in (24) is Hurwitz, $A-g h^{T}$ has one eigenvalue at the origin, and otherwise has all eigenvalues with negative real part. Furthermore, the product $A\left(A-g h^{T}\right)$ has one eigenvalue at the origin, and no eigenvalues on the negative real axis. Therefore, by Theorem 3 , there exists a positive definite $P$ such that $V(x)=x^{T} P x$ is a Lyapunov function for the system. This establishes stability for this system.

In fact, in this particular case, we can go further and establish asymptotic stability using the special structure involved. To do this we note the following.

Lemma 4.1 Consider $k_{\phi}(u)$ defined in (20). For any finite $K$ there exists an $\varepsilon_{K} \in(0,1]$, such that $\|x\| \leq K$ implies $k_{\phi}\left(h^{T} x\right) \geq \varepsilon_{K}$.

Proof The proof follows immediately from (20) by taking $\varepsilon_{K}=\min \left\{1, \frac{u_{\max }}{\|h\| K}\right\}$.

We then have the following result.

Theorem 4.1 Consider the system defined by (1) with $A, g, h, \sigma$ as defined in Section 4. Then for any finite initial condition, $x(0)$, it follows that $x(t) \rightarrow 0$.

Proof The proof follows from two main steps.

1. Lyapunov Stability From Theorem 3, we have Lyapunov stability and therefore for any finite initial condition, there exists a constant $K$ such that $\|x(t)\| \leq K$.

2. Asymptotic Stability Having established boundedness of the states, we now invoke Lemma 4.1. From (8), $A^{T} P+$ $P A=: Q$ is negative definite. Define $Q_{2}=\left(A-g h^{T}\right)^{T} P+P\left(A-g h^{T}\right) \leq 0$.

Then it can be shown that

$$
\begin{aligned}
\frac{d}{d t} x^{T} P x & =k_{\phi} x^{T} Q x+\left(1-k_{\phi}\right) x^{T} Q_{2} x \\
& \leq \varepsilon_{K} x^{T} Q x
\end{aligned}
$$

and asymptotic stability follows. 


\section{Concluding remarks}

In this note we have derived necessary and sufficient conditions for the existence of a common quadratic Lyapunov function for a pair of stable linear time-invariant systems whose system matrices are of the form $A$, $A-g h^{T}$, and where one of the matrices is singular. Future work will involve extending our results to non-quadratic stability criteria such as Popov.

\section{References}

[1] M. V. Kothare and M. Morari, "Multiplier theory for stability analysis of anti-windup control systems," $\mathrm{Au}$ tomatica, vol. 35, pp. 917-928, 1999.

[2] B. G. Romanchuck and M. C. Smith, "Incremental gain analysis of linear systems with bounded control and its application to the antiwindup problem," in Proceedings of the 35th Conference on Decision and Control, pp. 2942-2947, December 1996.

[3] M. J. Gomes da Silva and S. Tarbouriech, "Antiwindup design with guaranteed regions of stability: An lmi-based approach," IEEE Transactions on Automatic Control, vol. 50, pp. 106-111, January 2005.

[4] T. Loquen, S. Tarbouriech, and C. Prieur, "Stability analysis for reset systems with input saturation," in Proceedings of the 46th IEEE Conference on Decision and Control, pp. 3272-3277, December 2007.

[5] R. Shorten, M. Corless, K. Wulff, S. Klinge, and R. Middleton, "On the quadratic stability of singular switched systems." Submitted to IEEE Transactions on Automatic Control.

[6] S. Klinge, "Stability issues in distributed systems of vehicle platoons." Diplomarbeit Thesis, University of Magdeburg (http://www.steffiklinge.de/diplomarbeit_klinge_screen.pdf), 2008.

[7] H. Khalil, Nonlinear Systems: Third Edition. Prentice Hall, 2002.

[8] R. Shorten and K. S. Narendra, "On common quadratic Lyapunov functions for pairs of stable LTI systems whose system matrices are in companion form," IEEE Transactions on Automatic Control, vol. 48, no. 4, pp. 618-621, 2003.

[9] R. Shorten and C. King, "Spectral conditions for positive realness of SISO systems," IEEE Transactions on Automatic Control, vol. 49, pp. 1875-1877, Oct 2004.

[10] E. Zeheb and R. Shorten, "A note on spectral conditions for positive realness of SISO systems with strictly proper transfer functions," IEEE Transactions on Automatic Control, vol. 51, pp. 897-899, May 2006.

[11] R. Shorten, P. Curran, K. Wulff, and E. Zeheb, "A note on spectral conditions for positive realness of transfer function matrices," IEEE Transactions on Automatic Control, vol. 53, no. 5, pp. 1258-1261, 2008.

[12] Z. Bai and W. Freund, "Eigenvalue based characterisation and test for positive realness of scalar transfer functions," IEEE Transactions on Automatic Control, vol. 45, pp. 2396-2402, 2000.

[13] G. Muscato, G. Nunarri, and L. Fortuna, "Singular perturbation approximation of bounded real and positive real transfer matrices," in Proceedings of ACC, 1994.

[14] S. Boyd, L. El Ghaoui, E. Feron, and V. Balakrishnan, Linear Matrix Inequalities in System and Control Theory. Philadelphia: SIAM, 1994.

[15] A. Acikmese and M. Corless, "Stability analysis with quadratic Lyapunov functions: Some necessary and sufficient multiplier conditions," Systems and Control Letters, vol. 57, pp. 78-94, 2008. 